\title{
Beclomethasone/formoterol fixed combination for the management of asthma: patient considerations
}

\author{
Gabriele Nicolini' \\ Nicola Scichilone ${ }^{2}$ \\ Andrea Bizzi ${ }^{3}$ \\ Alberto $\mathrm{Papi}^{4}$ \\ Leonardo M Fabbri ${ }^{5}$ \\ 'Medical Department, Chiesi \\ Farmaceutici, Parma, Italy; ${ }^{2}$ University \\ of Palermo, DIMPEFINU, Palermo, \\ Italy; ${ }^{3}$ Department of Scientific Affairs, \\ Chiesi Farmaceutici, Parma, Italy; \\ ${ }^{4}$ Research Center on Asthma \\ and COPD, University of Ferrara, \\ Ferrara, Italy; ${ }^{5}$ Section of Respiratory \\ Diseases, University of Modena, \\ Modena, Italy
}

Correspondence: Leonardo M Fabbri

Department of Respiratory Diseases, University of Modena and ReggioEmilia,

Via del Pozzo 7I,I-4I 100 Modena, Italy

Tel +390594222198

Fax +390594224231

Email fabbri.leonardo@unimore.it

\begin{abstract}
Drugs for asthma and other chronic obstructive diseases of the lungs should be preferably delivered by the inhalation route to match therapeutic effects with low systemic exposure. Inhaled drugs are delivered to the lungs via different devices, mainly metered dose inhalers and dry powder inhalers, each characterized by specific inhaler technique and instructions for use. The patient-device interaction is part of the prescribed therapy and can have a relevant impact on adherence and clinical outcomes. The most suitable device should be considered for each patient to assure the correct drug intake and adherence to the prescribed therapy. The development of new drugs/devices in the past decades improved the compliance with inhaler and possibly drug delivery to the bronchi. The present review focuses on the recently developed beclomethasone/formoterol extrafine fixed combination and technical aspects of drug delivery to the lungs in patient's perspective.
\end{abstract}

Keywords: beclomethasone, formoterol, modulite, extrafine

\section{Introduction}

Inhaled therapy, which was developed within the last 50 years, is the cornerstone of asthma management and will remain so for the near future (GINA 2007). Although the effectiveness of inhaled therapy was reported as early as the beginning of the 20th century, it did not find widespread use until mid century, after the introduction of the first propellant gas dosing aerosols in the form of pressurized metered dose inhalers (pMDIs). Direct delivery of aerosolized asthma drugs to the lower airways is advocated to relieve airflow limitation and to suppress inflammation. In comparison with oral therapy, the inhaled route provides maximum benefit with minimum effective doses and few adverse effects. However, several factors, including drug formulation, delivery device, and the patient's skills, influence use of the inhaled route.

Currently available drugs for asthma treatment, including short- and long-acting bronchodilators alone or in combination and corticosteroids alone or in combination with bronchodilators, are all effective and reasonably safe (GINA 2007). The goal of asthma treatment, as defined in the recent guidelines update (GINA 2007; NAEPP 2007), is to reach and maintain asthma control, defined as minimal symptoms, no exacerbations, and no limitation of activities, together with normal lung function.

Most studies in recent years have shown that asthma can be well controlled in most patients with inhaled steroids alone or in combination with long-acting beta2 agonists (LABA) (O’Byrne et al 2001; Bateman et al 2004; Rabe et al 2006). In contrast, epidemiological studies show that, overall, asthma is not adequately treated (and thus not controlled) in patients in the "real world," probably because they behave differently from patients studied in randomized controlled trials: those patients are selected for their adherence to treatment and their ability to use the inhaler devices correctly. 
A recent publication (Cazzoletti et al 2007) evaluated asthma control across European countries; the prevalence of uncontrolled asthma ranged from $20 \%$ in Iceland to $67 \%$ in Italy, confirming previous observations (Rabe et al 2000; Partridge et al 2006).

The clinical benefit provided by inhaled therapy depends on several factors. From the patient's perspective, adherence to treatment and ability to inhale the drug seem to be the most important. Correct inhaler technique is a key factor in ensuring that the drug is deposited in the bronchi, where the pharmacological effect has to take place. Inadequate inhalation technique has been associated with loss of asthma control (Giraud and Roche 2002).

Despite the clinical importance of inhaled therapy, current guidelines lack consensus on recommended devices. In evidence-based guidelines for device selection (Brocklebank et al 2001; Dolovich et al 2005), all randomized controlled studies in which the same drug was delivered with different devices have been reviewed. None of the pooled metaanalyses showed a significant difference between devices in any efficacy outcome. The conclusions of these guidelines are that devices used for the delivery of bronchodilators and steroids can be equally efficacious, provided that patients use the correct technique for inhalation. Therefore, the selection criteria should be mainly related to the patient's age, preference, and ability to use the selected device correctly, rather than to a difference in clinical efficacy.

This review focuses on management issues in the treatment of asthma, provides an overview of pharmacology and rationale for the use of combinations, and discusses specific formulation characteristics and clinical efficacy of the beclomethasone/formoterol combination from patientfocused perspectives.

\section{Technical aspects of inhaled therapy}

The aim of inhaled therapy is to allow the medication to reach the site of structural and functional alterations of the airways. This can be obtained by satisfying the following conditions: (i) the drug delivery should allow the penetration of inhaled particles into the respiratory tract; (ii) the formulation should enable the drug to deposit along the respiratory tract; and (iii) the deposition of the drug should translate into functional and clinical benefits. Taken together, these conditions require careful attention to the choice of device (Brocklebank et al 2001).

The relative deposition pattern of the inhaled drug is the result of a complex interaction between the device, the aerosol formulation, and the patient's inhalation technique.
To allow the medication to reach the target site of action, a variety of hand-held inhalation devices has been introduced in clinical practice. The devices differ in terms of technical design, actuation (eg, inspiratory flow rate), composition (eg, characteristics of the propellant), vehicle, dose per inhalation, user-friendliness and cost.

The pMDI is the most widely used device (Virchow et al 2008). Since its introduction in 1956, it has been widely prescribed by health care professionals. The major limitation of the pMDI is the fast-moving aerosol, which increases the deposition of the drug in the pharynx, is responsible for local side effects (candidiasis, dysphonia) and does not contribute to clinical efficacy. The drug contained in the MDI canister can be formulated as a suspension or a solution, which have different properties in terms of delivery characteristics (particle size, plume velocity and duration) and userfriendliness. For years, MDI-formulated drugs have been mainly suspension formulations delivering large particles, thus reaching the lower airways at only $10 \%$ to $20 \%$ of the nominal dose, and propelled by chlorofluorocarbons (CFC) (Barnes 1995). A suspension formulation is obtained when the active drug is nonsoluble in the propellant and therefore remains in the form of solid powder inside the canister. To favor homogeneity of the drug concentration and consequent dose consistency and reproducibility, suspension formulations, unlike solutions, need to be shaken before inhalation to allow uniform distribution of solid powder particles of the drug. Unfortunately, $14 \%$ to $25 \%$ of patients do not shake the device properly (Van der Palen et al 1999), resulting in a variable amount of the drug being emitted for each puff. Moreover, suspension formulations need to be delivered with a relatively larger diameter of the orifice to avoid its blockage by the emitted drug. This leads to higher velocity and lower duration of the aerosol plume - resulting in increased drug deposition in the oropharynx - and requires coordination between actuation of the device and inhalation by the patient. In patients with poor coordination, the use of a spacer is often recommended to overcome this limitation.

Dry powder inhalers (DPIs) were introduced into clinical practice in the 1950s. Three types of DPIs are available with different handling instructions: single-dose (eg, Handihaler ${ }^{\circledR}$, Aerolizer $^{\circledR}$ ), multiple-dose (ie, Diskus ${ }^{\circledR}$ ), and reservoir (ie, Turbuhaler ${ }^{\circledR}$ ). DPIs do not need coordination, as the drug is not driven by the propellant but is delivered by the inhalation effort. For this reason, MDIs and DPIs require different techniques: slow and deep inhalation (MDIs) or quick, forceful, and deep inhalation (DPIs). The actuation of some DPI devices needs high inspiratory flow to ensure optimal 
drug delivery: both the particle size of emitted powder and the amount of drug suitable to reach the lower airways are dependent on the patient's peak inspiratory flow (Tarsin et al 2006). A recent study demonstrates that elderly patients are the least likely to generate high inspiratory flows, irrespective of the presence of airway obstruction (Janssens et al 2008).

Although DPI devices do not require coordination, they may be managed incorrectly by patients, resulting in a poor level of disease control; Wieshammer and Dreyhaupt (2008) reported that inhaler-specific error rates ranged from $9.1 \%$ to $53.1 \%$ and increased with age and with the severity of airway obstruction ( $\mathrm{p}<0.01)$. While MDIs share the same inhalation technique, each DPI device requires specific training, which may contribute to the increased risk of errors. Furthermore, the frequency of inhalation errors increased from $29 \%$ to $39 \%$ in adult asthmatics when multiple inhalers were prescribed (Van der Palen et al 1999).

In recent decades, possible adverse effects on health and the environment as a result of the destruction of the earth's ozone layer by CFC paved the way for the ban of these compounds under the Montreal Protocol (Hartung et al 2002; Newman, 1990). New propellants without ozone-damaging properties - hydrofluoroalkanes (HFA) - have been developed, and reformulated, environmentally friendly inhalers have been shown to be nontoxic, safe, and effective. Existing drugs can be formulated as HFA suspensions or HFA solutions (Figure 1).

The switch to HFA suspensions is possible when drugs that were suspended in CFC propellant are also suited to being suspended in HFA propellant while maintaining the same particle size, pattern of deposition, pharmacokinetics, efficacy, and safety profile. For example, fluticasone propionate in the new suspension with HFA propellant maintains the same dosage recommendations as the previous CFC formulation (NAEPP 2007).

The switch to HFA solutions - the reformulation of former CFC suspensions - is possible when the drug is soluble in HFA propellant (or co-solvents). In this case the solution is compatible with devices with smaller orifice diameters, leading to lower velocity and higher duration of the aerosol plume, thus facilitating the patient's coordination between actuation of the device and inhalation. Moreover, because the drug is dissolved in the propellant, solutions can be tailored to extrafine drug delivery, reducing the particle size of emitted aerosol and thereby allowing deeper penetration in the bronchial tree. Switching from a CFC pMDI to an HFA pMDI was well accepted by most patients in one study (Hartung et al 2002): the taste was described as good and sweet, and the softer and slower jet was appreciated.

\section{Recent developments in inhaled therapy devices: Modulite ${ }^{\circledR}$ technology}

The Chiesi Modulite platform technology allows the size and distribution of particles to be tailored for the specific airway targets. This is done through the manipulation of inhaled HFA-based solution pMDI formulations by acting
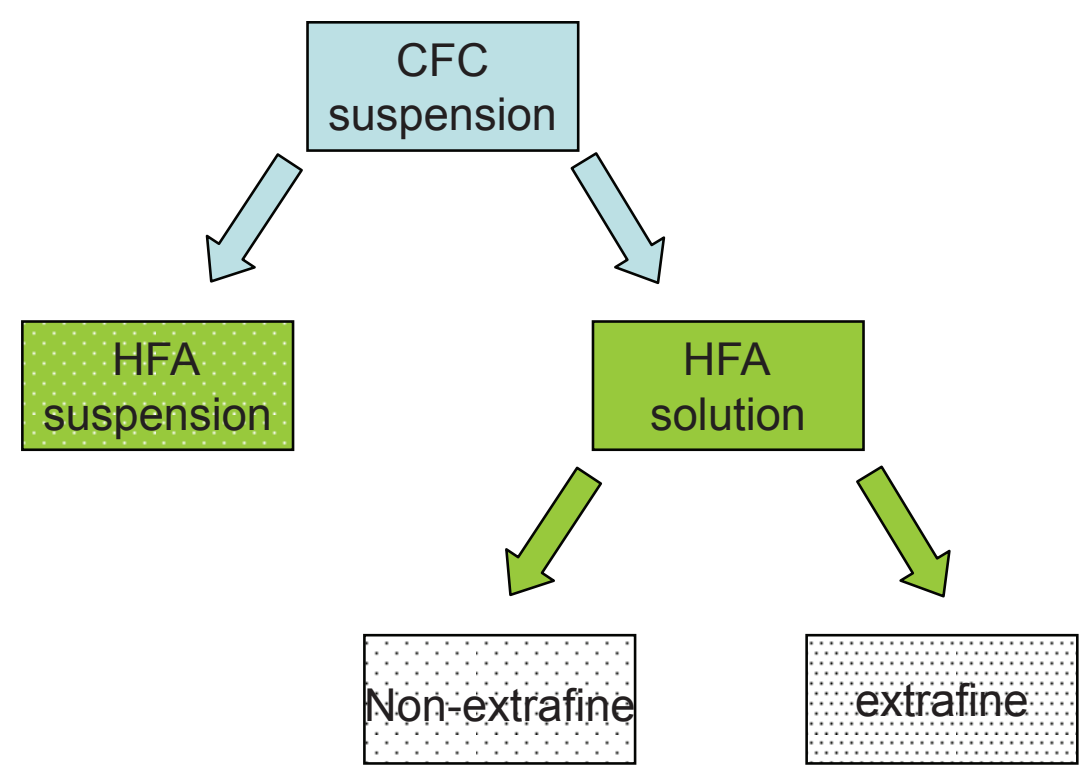

Figure I Reformulation from CFC-propelled to HFA-propelled pMDIs.

Abbreviations: HFA, hydrofluoroalkanes; CFC, chlorofluorocarbons; pMDI, pressurized metered dose inhaler.

Therapeutics and Clinical Risk Management 2008:4(5) 
on a number of variables, including formulation components and actuator orifice diameter (Acerbi et al 2007). This technology offers the opportunity to match the CFC-based pMDIs with the HFA MDIs, thus favoring the transition to environmentally friendly formulations. This kind of switch has been successfully achieved for corticosteroids such as beclomethasone and budesonide (Bousquet and Cantini 2002; Vastagh et al 2003). Moreover, by virtue of the solution formulation, this technology can be used to produce extrafine aerosols, such as the beclomethasone dipropionate/ formoterol fixed combination (BDP/F), which provides a homogeneous distribution of the two active drugs throughout the entire bronchial tree.

\section{Formulative characteristics of inhaled BDP/F extrafine fixed combination}

The BDP/F 100/6 HFA pMDI combination is an extrafine solution formulation in which the BDP dose is 2.5-fold lower than the conventional BDP CFC product (100 $\mu \mathrm{g}$ of BDP per actuation instead of $250 \mu \mathrm{g}$ of non-extrafine BDP). The reduction in BDP nominal dose, together with the extrafine particle size, allows a similar dose of the drug to reach the lower airways and less drug to be deposited in the upper airways, potentially improving the efficacy/safety ratio. The formoterol component in $\mathrm{BDP} / \mathrm{F}$ is not associated with nominal dose reduction (Dhillon and Keating 2006).

There is considerable literature available on BDP, covering all relevant outcomes in asthma treatment in both adults and children. In the only available study showing that inhaled corticosteroids (ICS) reduce asthma mortality (Suissa et al 2000), 93\% of the prescribed canisters contained lowdose BDP. Formoterol is the only fast, long-acting bronchodilator with dose-dependent effects (McGavin et al 2001) and well-documented efficacy and safety in obstructive diseases (Prenner 2007); it can also be used as a reliever medication in asthma, provided the patient is receiving regular ICS treatment (GINA 2007).

The rationale for developing an extrafine formulation lies in the fact that asthma is characterized by airway inflammation and remodeling in all parts of the airways, including small airways (Hamid et al 1997; Tulic et al 2001). Therefore, the optimized drug deposition that results from reduced particle size may lead to improved clinical benefits. Extrafine BDP has proved to be effective on candidate markers of peripheral airway inflammation (Gershman et al 1999; Hauber et al 2006).

Since extrafine particles are expected to improve drug delivery to the respiratory tree, the lung deposition and distribution pattern of BDP/F were recently assessed using a gamma-scintigraphic technique (Mariotti et al 2007). The average lung deposition was $34 \%$ relative to the nominal dose in healthy subjects and $31 \%$ in patients with asthma, suggesting that good delivery to the lung can be achieved regardless of the underlying pathophysiological condition (Figure 2). Table 1 compares lung deposition of different inhaler device categories.

Moreover, because the drug is delivered to both central and peripheral airways (Figure 3), a uniform treatment of inflammation and bronchoconstriction throughout the lung is expected.

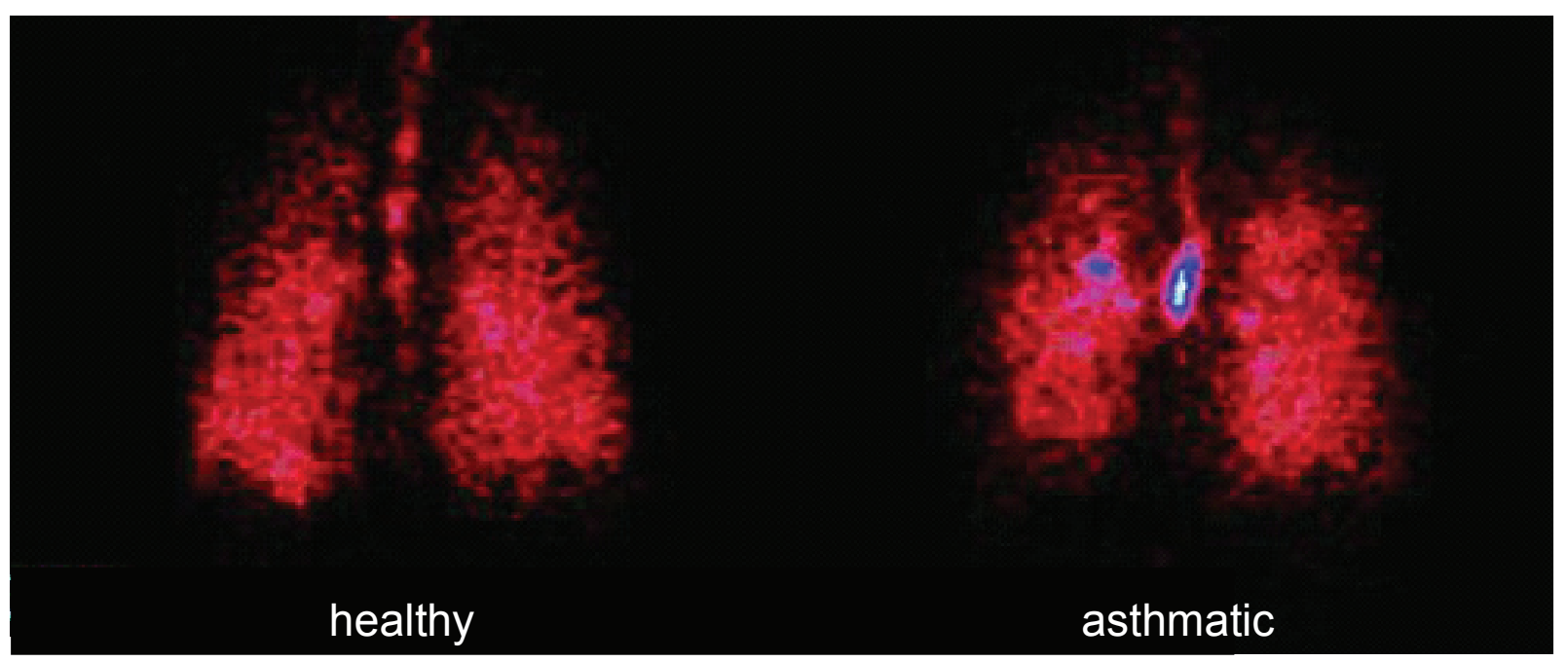

Figure 2 Scintigraphic images of deposition of BDP/F combination in a healthy subject and an asthmatic patient determined by $\gamma$-scintigraphy after inhalation of four shots of BDP/formoterol combination labelled with $99 \mathrm{mTc}$, a $\gamma$-emitting isotope (Mariotti et al 2007).

Abbreviation: BDP/F beclomethasone dipropionate/formoterol. 
Table I Lung deposition of different inhaler device categories (data from multiple studies)

\begin{tabular}{ll}
\hline & Lung deposition \% \\
\hline BDP/F pMDI (Mariotti et al 2007) & $31 \%$ \\
Conventional pMDIs (Selroos et al 1996) & $10 \%-20 \%$ \\
Conventional pMDIs plus spacer & $20 \%-30 \%$ \\
(Selroos et al I996) & \\
DPIs (Anderson 200I) & $15 \%-25 \%$ \\
\hline
\end{tabular}

Abbreviations: BDP/F, beclomethasone dipropionate/formoterol; pMDI, pressurized metered dose inhaler; DPI, dry powder inhaler.

\section{Rationale for ICS-LABA combinations}

ICS represent the mainstay of persistent asthma therapy and the addition of LABAs is the preferred option for patients whose asthma is not controlled with low doses of ICS alone (GINA 2007). The therapeutic value of ICS/LABA combinations is increased by the evidence suggesting that LABA and ICS mutually potentiate their effects when given in combination (Barnes 2002; Caramori et al 2006). Possible mechanisms of synergistic interactions include (i) the increase in expression of beta 2 receptors (ie, adrenergic receptors mediating smooth muscle relaxation in the airways) by increasing gene transcription induced by ICS leading to a greater number of receptors available for beta 2 agonists (Scott et al 1999), (ii) the increase in the nuclear localization of glucocorticoid receptors induced by beta 2 agonists potentiating the antinflammatory mechanisms of

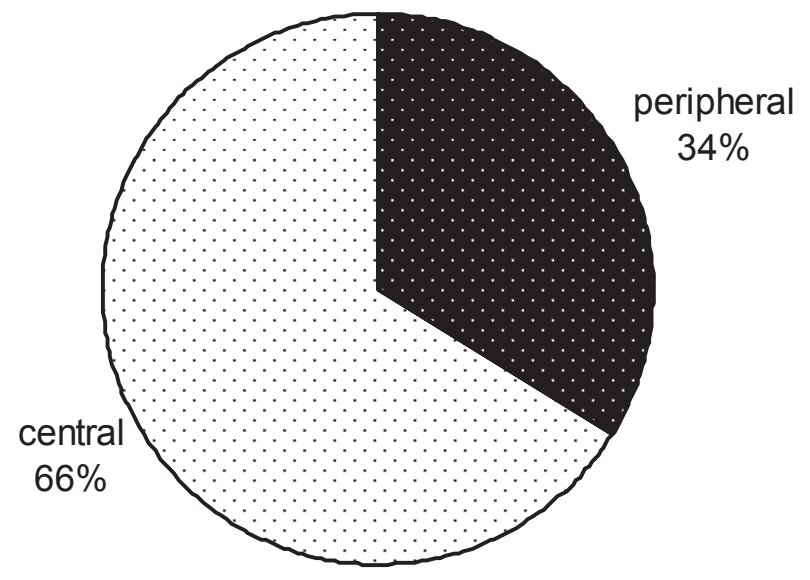

Figure 3 Percentage of central and peripheral lung deposition of radiolabeled BDP/F solution in 8 asthmatic patients. Lung contours for the assessment of regional deposition were assessed from an $81 \mathrm{mKrypton}$-ventilation scan after inhalation of 4 shots of BDP/formoterol combination labelled with $99 \mathrm{mTc}$, a $\gamma$-emitting isotope (adapted from Mariotti et al 2007).

Abbreviation: BDP/F, beclomethasone dipropionate/formoterol.
ICS (Eickelberg et al 1999), and (iii) the direct synergic action of both drugs on the release of inflammatory mediators promoting a more pronounced antiinflammatory effect (Caramori et al 2006).

The complementary effects of the combination of BDP and formoterol on the inflammatory process in patients with asthma were investigated in sputum-derived inflammatory cells in vitro (Profita et al 2005). Antiremodeling and antiproliferative effects were documented in human lung fibroblasts (Descalzi et al 2008). The findings of these studies indicate that the BDP/F combination induces a favorable pharmacodynamic interaction that can produce added benefits in terms of bronchodilation, antiinflammatory, antiremodeling, and antiproliferative effects.

\section{Clinical characteristics of the beclomethasone/formoterol (BDP/F) fixed combination}

Although beclomethasone and formoterol are well-known molecules of proven efficacy and safety, the clinical development of the BDP/F combination involved a series of clinical trials carried out in more than 1500 patients suffering from different levels of asthma severity. The pharmacokinetics profile of inhaled BDP/F extrafine fixed formulation was assessed in a 3-way crossover study (Poli et al 2006), which compared single doses of BDP/F 400/24 $\mu \mathrm{g}$, or an equipotent non-extrafine regimen of BDP and formoterol given via separate inhalers (1000 $\mu \mathrm{g}$ BDP non-extrafine and $24 \mu \mathrm{g}$ formoterol) or placebo. Plasma levels of the active metabolite of BDP, B-17-MP, in the first $30 \mathrm{~min}$ (area under the curve from time 0 to $30 \mathrm{~min}\left[\mathrm{AUC}_{0-30 \mathrm{~min}}\right]$ ), which can be considered an index of pulmonary deposition, were $86 \%$ greater with the fixed combination than with the separate inhalers. However, the $24 \mathrm{~h}$ systemic exposure of B-17-MP was 35\% lower with the fixed combination than with the separate inhalers. No difference between fixed combination and separate inhalers was shown for systemic exposure of formoterol.

In another study, asthmatic patients regularly treated for 4 weeks with BDP/F 100/6 $\mu \mathrm{g}$ as 2 inhalations twice daily (Singh et al 2008) were given 10 additional doses of $\mathrm{BDP} / \mathrm{F} 100 / 6 \mu \mathrm{g}$, formoterol $6 \mu \mathrm{g}$, or placebo on 3 different days (days 14, 21 and 28 of the study) to investigate the safety and effects of the two drugs. Compared with highdose $\mathrm{BDP} / \mathrm{F}$ and placebo, serum potassium significantly decreased only with high-dose formoterol. Changes in vital signs, including heart rate and blood pressure, corrected 
QT intervals, and plasma lactate levels were similar with high-dose BDP/F and high-dose formoterol but not considered clinically significant. This result demonstrates that, in patients with asthma, the administration of high cumulative doses of BDP/F in addition to maintenance therapy has a satisfactory tolerability profile and is at least as safe as the administration of high doses of formoterol alone.

A preliminary trial (Dhillon and Keating 2006; Rigamonti et al 2006) suggested that BDP extrafine $100 \mu \mathrm{g} /$ actuation, developed as a single agent, is clinically equivalent to BDP non-extrafine pMDI $250 \mu \mathrm{g} /$ actuation in patients with moderate asthma, demonstrating a 1:2.5 equivalence ratio between extrafine and non-extrafine BDP - a result already documented for a previously developed formulation (Vanden Burgt et al 2000).

The efficacy of the BDP/F fixed combination was then evaluated in a 3-month randomized controlled trial conducted in patients with moderate asthma who were still symptomatic despite receiving low-dose ICS - up to $500 \mu \mathrm{g} /$ day BDP or equivalent (Dhillon and Keating 2006; Bonnet-Gonod et al 2006a). BDP/F given as one inhalation twice daily proved to be more effective at improving lung function than a double equipotent dosage of BDP nonextrafine. A second investigation was carried out in patients with more severe asthma, documented by recurrent symptoms and impaired lung function despite treatment with up to $1000 \mu \mathrm{g} /$ day BDP or equivalent (Dhillon and Keating 2006; Bonnet-Gonod et al 2006b). In this setting, BDP/F given as 2 inhalations twice daily for 6 months showed improvement in peak expiratory flow (PEF) and forced expiratory volume in $1 \mathrm{~s}\left(\mathrm{FEV}_{1}\right)$ comparable with that of an equipotent non-extrafine regimen of BDP and formoterol administered via separate inhalers, and proved to be more effective than $1000 \mu \mathrm{g}$ /day BDP non-extrafine. Moreover, the $\mathrm{BDP} / \mathrm{F}$ fixed combination was superior to both BDP plus formoterol in separate inhalers and BDP monotherapy in terms of percentage of clinical measures of asthma control, suggesting that patients receiving extrafine $\mathrm{BDP} / \mathrm{F}$ may experience additional benefits that extend beyond improvements in pulmonary function. The percentage of patients with asthma exacerbations requiring oral steroids (percentage referred to patients with severe exacerbations) was lower in the group treated with the BDP/F fixed combination $(6.0 \%)$ than in those receiving BDP plus formoterol separately (12.1\%) or BDP alone (14.1\%). Mean morning serum cortisol levels at week 24 were significantly higher in the BDP/F group compared with baseline values, whereas no change was seen in the other two groups, suggesting that the extrafine fixed combination produced a lower inhibition of the hypothalamic-pituitary-adrenal axis compared with the other treatments. These results are in agreement with the low steroid intake that characterizes the BDP/F extrafine formulation. Finally, no clinically significant abnormalities of serum potassium and glucose were detected.

Two head-to-head clinical trials assessed the efficacy and tolerability of BDP/F vs budesonide/formoterol (BUD/F) and fluticasone propionate/salmeterol (FP/S), the currently marketed ICS/LABA fixed combinations (Papi et al 2007a, b). The two studies shared a similar study design. Subjects were allowed to enter the studies only if they had asthma symptoms and lung function $\left(\mathrm{FEV}_{1}\right)<80 \%$ of predicted despite receiving up to $1000 \mu \mathrm{g} /$ day BDP or equivalent. In the first trial (Papi et al 2007a), patients given BDP/F as 2 inhalations twice daily showed improvement in lung function, measured by morning pre-dose PEF, which was comparable with that with an equipotent regimen of BUD/F 200/6 $\mu$ g administered as 2 inhalations twice daily. Notably, when the speed of bronchodilation was evaluated as the change in $\mathrm{FEV}_{1}$ in the first 60 min after the morning dose on the first and last days of treatment, BDP/F demonstrated an onset of action equivalent to that of BUD/F. Both therapies were equally effective at improving asthma symptoms and increasing the percentage of days without the use of rescue medication (Table 2).

In the second trial (Papi et al 2007b), BDP/F was compared with fluticasone propionate/salmeterol (FP/S) pMDI $125 / 25 \mu \mathrm{g}$, both administered as 2 puffs twice daily. BDP/F demonstrated improvement in $\mathrm{PEF}$ and $\mathrm{FEV}_{1}$ comparable to that of FP/S when lung function was measured pre-dosing. The onset of bronchodilation, evaluated as a change in $\mathrm{FEV}_{1}$ in the first $60 \mathrm{~min}$ after the morning dose on the first and

Table 2 Percentage of symptom-free days and days with no intake of rescue salbutamol during head-to-head clinical trial of BDP/F vs BUD/F in asthma (Derived from data of Papi et al 2007a)

\begin{tabular}{lll}
\hline & $\begin{array}{l}\text { Symptom-free } \\
\text { days (\%) }\end{array}$ & $\begin{array}{l}\text { Days with no intake } \\
\text { of rescue salbutamol (\%) }\end{array}$ \\
\hline BDP/F & & 1.62 \\
Baseline & 2.61 & $52.07^{\mathrm{a}}$ \\
Last 2 weeks & $41.93^{\mathrm{a}}$ & \\
BUD/F & & 2.97 \\
Baseline & 3.52 & $48.92^{\mathrm{a}}$ \\
Last 2 weeks & $37.75^{\mathrm{a}}$ & \\
\hline
\end{tabular}

${ }^{a} p<0.001$ vs baseline, no significant difference between treatments. Values are the mean values of the last week of the run-in period (baseline) and the last 2 weeks of treatment.

Abbreviations: BDP/F, beclomethasone dipropionate/formoterol; BUD/F, budesonide/formoterol. 
last days of treatment, was significantly faster with BDP/F than with $\mathrm{FP} / \mathrm{S}$ owing to the pharmacodynamic properties of formoterol. This treatment can promote adherence in view of the fact that up to $90 \%$ of patients want to take treatments that provide immediate relief (Partridge et al 2006). For asthma control, assessed as days without symptoms and days without rescue use of salbutamol, comparable improvements were shown in the BDP/F and FP/S groups (Table 3). Notably, a greater improvement in forced vital capacity was shown in the $\mathrm{BDP} / \mathrm{F}$ group, possibly suggesting a greater effect on peripheral airways (van Aalderen et al 2007) (Figure 4).

In both studies no significant differences in the rate and severity of asthma exacerbations or in the incidence of adverse events were detected. Finally, even if these two comparative trials were powered for non-inferiority testing, the number of subjects evaluated give the studies $>85 \%$ power to show equivalence, with the original statistical assumptions (difference between treatments in morning $\mathrm{PEF}<20 \mathrm{~L} / \mathrm{min}$, with a standard deviation of $45 \mathrm{~L} / \mathrm{min}$ at an alpha level of 0.05 ). Because the $95 \%$ confidence intervals of the difference in the primary variable between treatments were within the pre-defined interval $( \pm 20 \mathrm{~L} / \mathrm{min})$, both studies would have supported an equivalence hypothesis (Papi et al $2007 \mathrm{a}, \mathrm{b})$.

\section{Patient considerations}

Drugs don't work in patients who don't take them.

C Everett Koop, MD

This sentence introduces an article published in the $N$ Engl $J$ Med (Osterberg and Blaschke 2005) that highlights the importance of a patient's adherence to prescribed medications and its contribution to clinical success. Indeed, patients are a

Table 3 Percentage of symptom-free days and days with no intake of rescue salbutamol during head-to-head clinical trial of BDP/F vs FP/S in asthma (Derived from data of Papi et al 2007b)

\begin{tabular}{lll}
\hline & $\begin{array}{l}\text { Symptom-free } \\
\text { days (\%) }\end{array}$ & $\begin{array}{l}\text { Days with no intake } \\
\text { of rescue salbutamol (\%) }\end{array}$ \\
\hline BDP/F & & 0 \\
$\quad$ Baseline & 0.25 & $60.65^{\mathrm{a}}$ \\
Last 2 weeks & $55.52^{\mathrm{a}}$ & \\
FP/S & & 1.15 \\
Baseline & 0.84 & $62.14^{\mathrm{a}}$ \\
Last 2 weeks & $54.25^{\mathrm{a}}$ & \\
\hline
\end{tabular}

${ }^{\mathrm{a}} \mathrm{P}<0.001$ vs baseline, no significant difference between treatments. Values are the mean values of the last week of the run-in period (baseline) and the last 2 weeks of treatment.

Abbreviations: BDP/F, beclomethasone dipropionate/formoterol; BUD/F, budesonide/formoterol. great part of the success or failure of therapy, and clinicians should always take this into account. It is often easier to respond to a patient's complaint that the medication is not working by simply writing a prescription for a higher dose or a different medication. Lack of efficacy with inhaled therapy can have several causes, not all related to pharmacological aspects of the drugs (Rubin 2004). For example, patients who cannot feel the medication working are more prone to non-adherence, particularly with ICS. This could explain why bronchodilators are sometimes overused by patients. The presence of the fast-acting bronchodilator formoterol in the $\mathrm{BDP} / \mathrm{F}$ combination can therefore contribute to improved adherence because patients are more willing to take treatments that provide immediate relief (Partridge et al 2006; Haahtela et al 2006).

Another aspect that may affectadherence to therapy is the patient's preference. A study to assess the acceptance of patients when changing from a CFC-driven device to a new HFA pMDI demonstrated that the HFA pMDI was the first choice of patients by virtue of past experience (Hartung et al 2002). The characteristics of the BDP/F extrafine combination may be helpful to some patients. For example, the slower velocity and longer duration of the plume may facilitate hand-breath coordination, thereby improving the patient's ability to use the device correctly.

Furthermore, unlike other pMDI suspensions, the BDP/F combination might not require down-titration of the therapeutic dose when used with a spacer. The more efficient lung deposition when a spacer is used may cause an increase in systemic drug exposure. Interestingly, BDP/F extrafine used with a spacer shows only slightly increased lung deposition, suggested by the peak in plasma drug concentration, with no significant difference in $24 \mathrm{~h}$ systemic exposure to the active metabolite of BDP (B-17-MP) or formoterol (Figure 5) (Poli et al 2007). Finally, the solution formulation eliminates the need to shake the device before inhalation, which is mandatory for suspensions and is commonly overlooked by patients, resulting in variable dose delivery.

Some clinical improvements have been demonstrated for the $\mathrm{BDP} / \mathrm{F}$ combination that could be due to the properties of the extrafine solution formulation. First, whereas a higher adherence to therapy is expected for all fixed combinations because of the convenience of a single inhaler instead of two, BDP/F is the first combination to show significant improvement in asthma control measures as compared to separate inhalers (Bonnet-Gonod et al 2006b; Fabbri et al 2008). Second, the reduced BDP dose - a consequence of the improved drug delivery of the formulation - allows the use of lower doses 


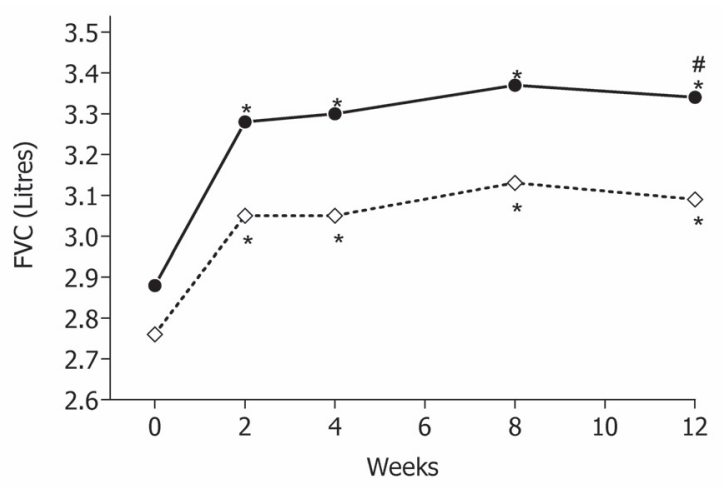

Figure 4 Forced vital capacity (FVC, L) measured at clinics in the two groups (BDP/F: -- $;$ FP/S: $\diamond---\diamond) ;{ }^{*}<<0.001$ vs baseline; \#p $=0.040$ between treatments. Derived from data of Papi et al 2007b.

Abbreviation: BDP/F, beclomethasone dipropionate/formoterol; FP/S, fluticasone propionate/salmeterol.

of ICS with resulting advantages in safety and adherence. Clinical effects of ICS are evident even at low doses, because the dose-response curve is steep for low doses and rapidly flattens, whereas systemic side effects are dose dependent. The safety profile of the BDP/F combination seems to be improved, as suggested by a lower systemic exposure to B-17-MP, which is potentially responsible for systemic side effects. Lower steroid exposure was demonstrated for $24 \mathrm{~h}$ serum cortisol in the pharmacokinetics study and for morning serum cortisol in the 6-month study. As long as one of the reasons for poor adherence to ICS treatment is that patients are concerned about side effects (Rubin 2004), the lower dose of BDP in this formulation could help to improve adherence.

Together with the slower plume velocity and the reduced particle size of the emitted drug, the lower nominal dose of $\mathrm{BDP}$ in the $\mathrm{BDP} / \mathrm{F}$ combination leads to a reduction in

\section{B-17-MP AUC ${ }_{0-\mathrm{t}}$}

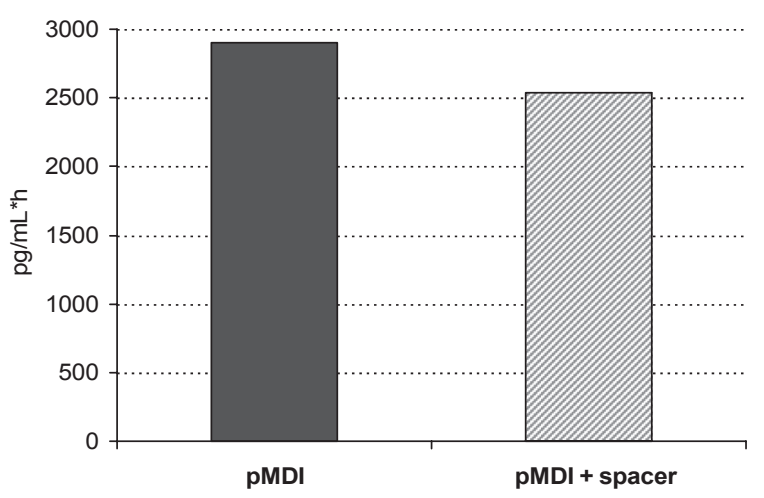

the amount of drug deposited in the oropharynx because of inertial impact. This could be an advantage, because the drug deposited in the upper airways is responsible for local side effects such as hoarseness, dysphonia, and candidiasis (Roland et al 2004).

Histological and functional evidence suggests that the small airways are an important site of airway inflammation in asthma. Reaching the more peripheral airways could therefore translate into higher efficacy of the inhaled therapy (Pritchard and Sharma 2001). For patient-centered outcomes, clinically important improvements in quality of life (evaluated by the Asthma Quality of Life Questionnaire score) were observed after 1 year of treatment with extrafine BDP compared with equipotent (double) doses of non-extrafine CFC BDP. Conventional clinical indexes of pulmonary function and asthma control were similar in the two groups (Juniper et al 2002). Recently, a subgroup analysis of an open label study indicated that patients receiving LABAs and treated with extrafine BDP had significantly greater improvement in asthma control, the ultimate measure of therapeutic efficacy, compared with fluticasone DPI. This suggests that there are potential advantages to extrafine aerosols as part of the optimization of antiinflammatory treatment (Molimard et al 2005).

\section{Conclusion}

When a new drug is marketed, clinicians may be concerned that it will have lower efficacy or a higher risk of adverse events than already available drugs in the same therapeutic indication. Because BDP and formoterol are not new chemical entities, they are not likely to expose patients to the risk of unexpected or unknown side effects.

Figure 5 Area under the curve from time 0 to the last detectable drug concentration value (AUC $\mathrm{C}_{0-\mathrm{t}}$ ) for beclomethasone- 17-monopropionate (B-I7-MP) (left) and formoterol (right), after 4 inhalations of BDP/F I00/6 $\mu$ g, with standard actuator (pMDI) or standard actuator with Aerochamber Plus (pMDI + spacer). From Poli et al 2007. Abbreviations: BDP/F, beclomethasone dipropionate/formoterol; pMDI, pressurized metered dose inhaler. 
Despite reassuring data on this topic (Allen et al 1994; Inoue et al 1999; Leone et al 2003; Pedersen 2006), a patient's concern about a medication's side effects, especially with corticosteroids, can be a cause of non-adherence and a resulting lack of efficacy. Issues about the safety of ICS, especially for long-term treatments, have been debated for years. However, all asthma guidelines now recommend low doses of ICS as the mainstay of therapy, since it has been strongly proved that the benefits largely overcome the risks, even in children (Becker et al 2005; British Thoracic Society 2007; GINA 2007; NAEPP 2007). The clinical effects of the BDP/F combination are in line with current guidelines that recommend adding a LABA for those patients whose asthma is uncontrolled after an introductory dose of ICS, instead of increasing the dose of ICS. The efficacy and safety of BDP/F are equivalent to those of already available combinations (Papi et al 2007a, b). Therefore, the BDP/F fixed combination seems to be a valuable option in the treatment of asthma, satisfying patients' needs for improved efficacy, improved safety profile and ease of use.

\section{Disclosures}

Dr Nicolini and Dr Bizzi are employees of Chiesi Farmaceutici, the commercial entity that produces BDF/F combination.

Prof Papi has served as a consultant to Chiesi Farmaceutici and GlaxoSmithKline; has been paid lecture fees by AstraZeneca, Chiesi Farmaceutici, Boehringer Ingelheim, GlaxoSmithKline, and Merck Sharp and Dohme; and has received grant support from AstraZeneca, Chiesi Farmaceutici, Boehringer Ingelheim, Merck Sharp and Dohme, and the Italian Ministry for University and Research.

Dr Scichilone has been paid lecture fees by Chiesi Farmaceutici, GlaxoSmithKline, Pfizer, and Sigma Tau, and has received grant support from Boehringer Ingelheim and Chiesi Farmaceutici.

Prof Fabbri has served as a consultant to Altana Pharma, AstraZeneca, Boehringer Ingelheim, Chiesi Farmaceutici, GlaxoSmithKline, Merck Sharp and Dohme, Novartis, Roche, and Pfizer; has been paid lecture fees by Altana Pharma, AstraZeneca, Boehringer Ingelheim, Chiesi Farmaceutici, GlaxoSmithKline, Merck Sharp and Dohme, Novartis, Roche, and Pfizer; and has received grant support from Altana Pharma, AstraZeneca, Boehringer Ingelheim, Menarini, Miat, Schering Plough, Chiesi Farmaceutici, GlaxoSmithKline, Merck Sharp and Dohme, UCB Pharma, Pfizer, Italian Ministry of Health, and the Italian Ministry for University and Research.

\section{References}

Acerbi D, Brambilla G, Kottakis I. 2007. Advances in asthma and COPD management: delivering CFC-free inhaled therapy using Modulite technology. Pulm Pharmacol Ther, 20:290-303.

Allen DB, Mullen M, Mullen B. 1994. A meta-analysis of the effect of oral and inhaled corticosteroids on growth. J Allergy Clin Immunol, 93:967-76.

Anderson PJ. 2001. Delivery options and devices for aerosolized therapeutics. Chest, 120(3 Suppl):89S-93S.

Barnes PJ. 1995. Inhaled glucocorticoids for asthma. $N$ Engl J Med, 332:868-75.

Barnes PJ. 2002. Scientific rationale for inhaled combination therapy with long-acting beta2-agonists and corticosteroids. Eur Respir J, 19:182-91.

Bateman ED, Boushey HA, Bousquet J, et al. 2004. Can guideline-defined asthma control be achieved? The Gaining Optimal Asthma ControL study. Am J Respir Crit Care Med, 170:836-44.

Becker A, Lemière C, Bérubé D, et al. 2005. Summary of recommendations from the Canadian Asthma Consensus guidelines, 2003. CMAJ, 173(6 Suppl):S3-11.

Bonnet-Gonod F, Kottakis I, Ballabio M, et al. 2006a. Superior efficacy of a low daily dose of a new fixed combination of beclomethasone dipropionate/formoterol pMDI compared to increased daily dose of BDP in moderate persistent asthma: a 3 month clinical study (abstract). Eur Respir J, 28(Supp1 50):P1237.

Bonnet-Gonod F, Kottakis I, Hofman T, et al. 2006b. Beclomethasone dipropionate/formoterol in a single inhaler improves lung function and clinically meaningful outcomes in moderate to severe asthma (abstract). Eur Respir J, 28(Suppl 50):P1230.

Bousquet J, Cantini L. 2002. Clinical studies in asthmatics with a new non-extrafine HFA formulation of beclometasone dipropionate (BDP Modulite). Respir Med, 96(Suppl D):S17-27.

British Thoracic Society. 2007. British guideline on the management of asthma. A national clinical guideline. Edinburgh (Scotland): Scottish Intercollegiate Guidelines Network (SIGN).

Brocklebank D, Ram F, Wright J, et al. 2001. Comparison of the effectiveness of inhaler devices in asthma and chronic obstructive airways disease: a systematic review of the literature. Health Technol Assess, 5:1-149.

Caramori G, Ito K, Papi A, et al. 2006. Interactions between long-acting B2-agonists and glucocorticoids. Drug Discov Today Ther Strateg, 3:261-8

Cazzoletti L, Marcon A, Janson C, et al. 2007. Asthma control in Europe: a real-world evaluation based on an international population-based study. J Allergy Clin Immunol 120:1360-7.

Descalzi D, Folli C, Nicolini G, et al. 2008. Anti-proliferative and antiremodelling effect of beclomethasone dipropionate, formoterol and salbutamol alone or in combination in primary human bronchial fibroblasts. Allergy, 63:432-7.

Dhillon S, Keating GM 2006. Beclomethasone dipropionate/formoterol: in an HFA-propelled pressurised metered-dose inhaler. Drugs, 66:1475-83.

Dolovich MB, Ahrens RC, Hess DR, et al. 2005. Device selection and outcomes of aerosol therapy: Evidence-based guidelines: American College of Chest Physicians/American College of Asthma, Allergy, and Immunology. Chest, 127:335-71.

Eickelberg O, Roth M, Lörx R, et al. 1999. Ligand-independent activation of the glucocorticoid receptor by beta2-adrenergic receptor agonists in primary human lung fibroblasts and vascular smooth muscle cells. J Biol Chem, 274:1005-10.

Fabbri LM, Nicolini G, Olivieri D, et al. 2008. Inhaled beclometasone dipropionate/formoterol extrafine fixed combination in the treatment of asthma: evidence and future perspectives. Expert Opin Pharmacother, 9:479-90.

Gershman NH, Liu H, Wong HH, et al. 1999. Fractional analysis of sequential induced sputum samples during sputum induction: evidence that different lung compartments are sampled at different time points. J Allergy Clin Immunol, 104:322-8. 
GINA (Global Initiative on Asthma), National Institutes of Health, National Heart Lung and Blood Institute, NHLBI/WHO Workshop Report. Global strategy for asthma management and prevention. NHLBI/WHO workshop report no. 02-3659, January 1995, updated 2007.

Giraud V, Roche N. 2002. Misuse of corticosteroid metered-dose inhaler is associated with decreased asthma stability. Eur Respir J, 19:246-51.

Haahtela T, Tamminen K, Malmberg LP, et al. 2006. Formoterol as needed with or without budesonide in patients with intermittent asthma and raised NO levels in exhaled air: a SOMA study. Eur Respir J, 28:748-55.

Hamid Q, Song Y, Kotsimbos TC, et al. 1997. Inflammation of small airways in asthma. J Allergy Clin Immunol, 100:44-51.

Hartung TK, Allbutt H, Dewar M, et al. 2002. Moving from CFC aerosol to HFA aerosol or dry powder inhalers: what do patients think? Respiration, 69:314-9.

Hauber H, Taha R, Bergeron C, et al. 2006. Effects of hydrofluoroalkane and dry powder-formulated corticosteroids on sputum inflammatory markers in asthmatic patients. Can Respir J, 13:73-8.

Inoue T, Doi S, Takamatsu I, et al. 1999. Effect of long-term treatment with inhaled beclomethasone dipropionate on growth of asthmatic children. $J$ Asthma, 36:159-64.

Janssens W, VandenBrande P, Hardeman E, et al. 2008. Inspiratory flow rates at different levels of resistance in elderly COPD patients. Eur Respir J. 31:78-83.

Juniper EF, Price DB, Stampone PA, et al. 2002. Clinically important improvements in asthma-specific quality of life, but no difference in conventional clinical indexes in patients changed from conventional beclomethasone dipropionate to approximately half the dose of extrafine beclomethasone dipropionate. Chest, 121:1824-32.

Leone FT, Fish JE, Szefler SJ, et al. 2003. Systematic review of the evidence regarding potential complications of inhaled corticosteroid use in asthma: collaboration of American College of Chest Physicians, American Academy of Allergy, Asthma, and Immunology, and American College of Allergy, Asthma, and Immunology. Chest, 124:2329-40.

Mariotti F, Poli G, Acerbi D, et al. 2007 Lung deposition of BDP/formoterol HFA pMDI in healthy volunteers, asthmatic and COPD patients [abstract]. Poster presented at the VIII UIP National congress, Florence, 4-7 December 2007.

McGavin JK, Goa KL, Jarvis B. 2001. Inhaled budesonide/formoterol combination. Drugs, 61:71-8.

Molimard M, Martinat Y, Rogeaux Y, et al. 2005. Improvement of asthma control with beclomethasone extrafine aerosol compared to fluticasone and budesonide. Respir Med, 99:770-8.

NAEPP (National Asthma Education and Prevention Program). 2007. Expert Panel Report 3. Guidelines for the Diagnosis and Management of Asthma - Summary Report 2007. J Allergy Clin Immunol, 120(5 Suppl):S94-138.

Newman SP. 1990. Metered dose pressurized aerosols and the ozone layer. Eur Respir J. May; 3(5):495-7.

O’Byrne PM, Barnes PJ, Rodriguez-Roisin R, et al. 2001. Low dose inhaled budesonide and formoterol in mild persistent asthma: the OPTIMA randomized trial. Am J Respir Crit Care Med, 164:1392-7.

Osterberg L, Blaschke T. 2005. Adherence to medication. N Engl J Med, 353:487-97.

Papi A, Paggiaro PL, Nicolini G, et al. 2007a. Beclomethasone/formoterol versus budesonide/formoterol combination therapy in asthma. Eur Respir J, 29:682-9.

Papi A, Paggiaro PL, Nicolini G, et al. 2007b. Beclomethasone/formoterol versus fluticasone/salmeterol inhaled combination in moderate to severe asthma. Allergy, 62:1182-8.

Partridge MR, van der Molen T, Myrseth SE, et al. 2006. Attitudes and actions of asthma patients on regular maintenance therapy the INSPIRE study. BMC Pulm Med, 6:13.

Pedersen S. 2006. Clinical safety of inhaled corticosteroids for asthma in children: an update of long-term trials. Drug Saf, 29:599-612.
Poli G, Acerbi D, Nollevaux F. 2006. Pharmacokinetics and pharmacodynamics of a new beclomethasone dipropionate and formoterol CFCfree fixed combination in healthy volunteers (abstract). Eur Respir $J$, 28(Suppl 50):P3869.

Poli G, Acerbi D, Rusca A. 2007. Pharmacokinetics and lung bioavailability of Foster using the standard actuator or the Aerochamber Plus spacer (abstract). Eur Respir J, 30(Abstract Suppl):P2117.

Prenner BM. 2007. Formoterol dry-powder inhaler for the treatment of asthma and chronic obstructive pulmonary disease. Expert Opin Pharmacother, 8:3069-84.

Pritchard JN, Sharma RK. 2001. Introduction: inhaled drug delivery. $J$ Aerosol Med, 14(Suppl 1):S1-2.

Profita M, Gagliardo R, Di Giorgi R, et al. 2005. Biochemical interaction between effects of beclomethasone dipropionate and salbutamol or formoterol in sputum cells from mild to moderate asthmatics. Allergy, 60:323-9.

Rabe KF, Vermeire PA, Soriano JB, et al. 2000. Clinical management of asthma in 1999: the Asthma Insights and Reality in Europe (AIRE) study. Eur Respir J, 16:802-7.

Rabe KF, Atienza T, Magyar P, et al. 2006. Effect of budesonide in combination with formoterol for reliever therapy in asthma exacerbations: a randomised controlled, double-blind study. Lancet, 368:744-53.

Rigamonti E, Kottakis I, Pelc M, et al. 2006. Comparison of a new extrafine beclomethasone dipropionate HFA 134a-formulated pMDI with a standard BDP CFC pMDI in adults with moderate persistent asthma (abstract). Eur Respir J, 28(Suppl 50):P1236.

Roland NJ, Bhalla RK, Earis J. 2004. The local side effects of inhaled corticosteroids: current understanding and review of the literature. Chest, 126:213-9.

Rubin BK. 2004. What does it mean when a patient says, "my asthma medication is not working"? Chest, 126:972-81.

Scott MG, Swan C, Wheatley AP, et al. 1999. Identification of novel polymorphisms within the promoter region of the human beta 2 adrenergic receptor gene. Br J Pharmacol, 126:841-4.

Selroos O, Pietinalho A, Riska H. 1996. Delivery devices for inhaled asthma medication. Clinical implications of differences in effectiveness. Clin Immunother, 4:273-99.

Singh D, Piccinno A, Borrill Z, et al. 2008. Tolerability of high cumulative doses of the HFA modulite beclomethasone dipropionate/formoterol combination inhaler in asthmatic patients. Pulm Pharmacol Ther, 21:551-7.

Suissa S, Ernst P, Benayoun S, et al. 2000. Low-dose inhaled corticosteroids and the prevention of death from asthma. N Engl J Med, 343:332-6.

Tarsin WY, Pearson SB, Assi KH, et al. 2006. Emitted dose estimates from Seretide Diskus and Symbicort Turbuhaler following inhalation by severe asthmatics. Int J Pharm, 316:131-7.

Tulic MK, Christodoulopoulos P, Hamid Q. 2001. Small airway inflammation in asthma. Respir Res, 2:333-9.

Van Aalderen WM, Price D, De Baets FM, et al. 2007. Beclometasone dipropionate extrafine aerosol versus fluticasone propionate in children with asthma. Respir Med, 101:1585-93.

Vanden Burgt JA, Busse WW, Martin RJ, et al. 2000. Efficacy and safety overview of a new inhaled corticosteroid, QVAR (hydrofluoroalkanebeclomethasone extrafine inhalation aerosol), in asthma. J Allergy Clin Immunol, 106:1209-26.

Van der Palen J, Klein JJ, van Herwaarden CL, et al. 1999. Multiple inhalers confuse asthma patients. Eur Respir J, 14:1034-7.

Vastagh E, Kuna P, Calistruc P, et al. 2003. Efficacy and safety of inhaled budesonide delivered once or twice daily via HFA-134a in mild to moderate persistent asthma in adult patients. Comparison with budesonide CFC. Respir Med, 97(Suppl D):S20-8.

Virchow JC, Crompton GK, Dal Negro R, et al. 2008. Importance of inhaler devices in the management of airway disease. Respir Med, 102:10-9.

Wieshammer S, Dreyhaupt J. 2008. Dry powder inhalers: which factors determine the frequency of handling errors? Respiration, 75:18-25. 